\title{
Retrieval of Cloud Water Variables by 1D-Var Algorithm
}

\author{
Qun-Bo Huang ${ }^{1 *}$, Wei-Min Zhang ${ }^{2}$, Bai-Nian Liu ${ }^{3}$, De Xing ${ }^{4}$, Xiang Xing ${ }^{5}$, Teng-Ling Luo ${ }^{6}$, Wei-Feng Wang ${ }^{7}$ \\ Xiao-Gang Zhang ${ }^{8}$
}

${ }^{1 *}$ Academy of Ocean Science and Engineering National University of Defense Technology, Changsha, China

$1^{*}$ College of Computer, National University of Defense Technology, Changsha, China

$1^{1 *}$ Weather Center of PLA Air Force, PLA Air Force, Beijing, China

${ }^{2}$ Academy of Ocean Science and Engineering National University of Defense Technology, Changsha, China

${ }^{3}$ Academy of Ocean Science and Engineering National University of Defense Technology, Changsha, China

${ }^{3}$ College of Computer, National University of Defense Technology, Changsha, China

${ }^{4}$ Academy of Ocean Science and Engineering National University of Defense Technology, Changsha, China

${ }^{4}$ College of Computer, National University of Defense Technology, Changsha, China

${ }^{5}$ Academy of Ocean Science and Engineering National University of Defense Technology, Changsha, China

${ }^{5}$ College of Computer, National University of Defense Technology, Changsha, China

${ }^{6}$ Academy of Ocean Science and Engineering National University of Defense Technology, Changsha, China

${ }^{6}$ College of Computer, National University of Defense Technology, Changsha, China

${ }^{7}$ Weather Center of PLA Air Force, PLA Air Force, Beijing, China

${ }^{8}$ Meteorological Observatory, Fourteen Suboffice of Jiuquan, No. 435, Jiuquan, China

1*hqb09@163.com, 813830140531@163.com

\begin{abstract}
Data assimilation of satellite observations in cloud and rain regions is a worldwide problem. In the paper, we designed the background field and their error covariance of three cloud water variables (cloud cover, cloud liquid water and cloud ice water) at first. The experiment results demonstrate that 1D-Var (One-dimensional variational data assimilation) can retrieve the three variables efficiently. The cloud liquid water and cloud ice water can be obviously improved by 1D-Var. However, the retrieved effect of low cloud cover is still to be improved. It has the positive potential to assimilate the satellite observations in cloud and rain regions.
\end{abstract}

\section{Introduction}

Clouds and precipitation are produced under certain weather conditions, which are related to the majority of the important weather phenomena (such as thunderstorms, hail, tornadoes, heavy rain and so on). The assimilation of satellite data in cloud and rain regions will improve the accuracy of numerical weather forecast, and which is currently a hotspot problem in the world.

Chevallier and Bauer simulated satellite observations under cloud and rain condition by RTTOV, and then compared with Special Sensor Microwave/Imager (SSM/I) observations [1]. Ma et al. analyzed the error of simulated brightness temperature and the sensitivity to cloud parameters (cloud cover and cloud height) of the infrared instrument channels on NOAA14 [2]. Ding et al. used the RTTOV8.7 to test the simulated effect of the brightness temperature of $\mathrm{HIRS} / 3$ in the cloudy regions existing a typhoon [3].

$1 \mathrm{D}-$ Var is typically used as a retrieval and preprocessing method due to its algorithmic simplicity and easy to control [4]. Huang studied the basic principles of the 1D+4D-Var two-step method and designed a
1D+4D-Var system process which is employed to assimilate the cloud and rain affected SSM/I data [5].

Based on the 1D-Var software developed by ECMWF, This paper presents a method of retrieving the cloud water variables by satellite observations. We firstly investigate the fundamental problems of cloud and rain data assimilation. Section II describes the algorithm process of $1 \mathrm{D}-$ Var. Section III designs the background field and the error covariance matrix of cloud water variables. Section IV shows and analyzes the numerical experiment results. Finally, conclusions and plans for future work are provided in section $\mathrm{V}$.

\section{1D-Var algorithm}

According to the Bayesian and variational theory, the cost function in 1D-Var can be expressed as:

$$
J(\mathbf{x})=\frac{1}{2}\left(\mathbf{x}-\mathbf{x}_{b}\right)^{T} \mathbf{B}^{-1}\left(\mathbf{x}-\mathbf{x}_{b}\right)+\frac{1}{2}\left[\mathbf{y}^{0}-H(\mathbf{x})\right]^{T} \mathbf{R}^{-1}\left[\mathbf{y}^{0}-H(\mathbf{x})\right]
$$

Where the background error is assumed to not dependent on the observation error, and which are Gaussian distribution. $\mathrm{x}$ is background profile, $\mathrm{B}$ and $\mathrm{R}$ 
are the background error covariance matrix and the observation error covariance matrix, $y$ is satellite observation. $\mathrm{H}(\mathrm{x})$ represents the radiance which is simulated by the forward model of RTTOV, where $\mathrm{x}$ is the input parameter of RTTOV.

By minimizing the cost function (1), the optimal state variables (i.e. analysis field) can be obtained.

$$
\mathbf{x}_{a}=\arg \min _{\mathbf{x}}\left(J\left(\mathbf{x}_{b}\right)\right)
$$

Minimization algorithms are generally the conjugate gradient method or quasi-Newton iteration method [6].

\section{Background Field And Matrix B}

The process of minimization calculation is controlled by the background and the observation error covariance matrix. The background error covariance matrix $\mathrm{B}$ is an essential parameter for variational analysis, which is utilized to control the vertical distribution and transmission of observation information. The vertical correlation conceals in the matrix B (the row of which is usually called as a structural function) is then transmitted to the analysis field. If background error can't clear in the retrieved/analysis field, it will lead to completely distorted analysis information, and may even have a bigger error than that of the background state.

The real matrix B is too difficult to learn, so we usually get it by statistical method. However, if the statistical sample is not sufficient, the theoretical calculation becomes an alternative tool, and this is the idea of the paper.

Assuming that background field consists of three variables $\left(t, q\right.$ and ${ }^{o_{3}}$ ) which are not related to each other. The covariance matrix can be expressed as a block diagonal matrix $B$ which consists of three sub-

$$
\begin{aligned}
& \text { matrices }{ }^{\mathbf{B}_{t},}{ }^{\mathbf{B}_{q}} \text { and } \mathbf{B}_{o 3} \text { : } \\
& \mathbf{B}=\left[\begin{array}{ccc}
\mathbf{B}_{t} & \mathbf{O} & \mathbf{O} \\
\mathbf{O} & \mathbf{B}_{q} & \mathbf{O} \\
\mathbf{O} & \mathbf{O} & \mathbf{B}_{o 3}
\end{array}\right]
\end{aligned}
$$

where the three sub-matrices represent the vertical correlation among the three variables, and should be banded because of the large correlation between the adjacent atmospheric pressure levels.

For cloud and rain affected satellite observations, we mainly consider the liquid and ice water content in clouds in the atmosphere which are determined by three variables: cloud cover, cloud liquid water content and cloud ice water content.

For cloud cover, the background field is:

$$
\mathbf{x}_{c c}(k)= \begin{cases}1.0 \times 10^{-4} & ; k>\text { itrop } \\ 0 & ; k<\text { itrop }\end{cases}
$$

Where $k$ is the atmospheric pressure levels, ${ }^{i t r o p}$ is the level number of the tropopause where the lowest temperature level locates between the $35 \mathrm{hPa}$ and $500 \mathrm{hPa}$. If the pressure level is less than itrop, which is above the tropopause; If the pressure level is bigger than itrop, which is below the tropopause.

For cloud liquid water content, the background field is:

$$
\mathbf{x}_{c l w}(k)= \begin{cases}2.0 \times 10^{-6} & ; k>\text { itrop } \text { and temperture }>260 \mathrm{~K} \\ 0 & ; k<\text { itrop and temperature } \leq 260 \mathrm{~K}\end{cases}
$$

For cloud ice water content, the background field is:

$$
\mathbf{x}_{c i w}(k)= \begin{cases}2.0 \times 10^{-6} & ; k>\text { itrop } \text { and temperture }>274 \mathrm{~K} \\ 0 & ; k<\text { itrop and temperature } \leq 274 \mathrm{~K}\end{cases}
$$

The design of background error covariance B needs to consider the correlation between the vertical pressure levels, and meeting the conditions as follows: (1) the levels below the tropopause and above the tropopause are independent of each other, (2) the levels above the tropopause are independent of each other, (3) the levels below the tropopause are only related to the pressure difference between the pressure levels. If the correlation is less than 0.01 , it should be set to zero. On this basis, the spatial correlation matrix can be derived:

$$
S C_{i j}= \begin{cases}0 & ; i, j<i \text { trop, and } i \neq j \\ 1 & ; i, j<i \text { trop, and } i=j \\ 0 & ; i<\text { itrop \& } j>\text { itrop } ; \text { or } i>\text { itrop } \& j<\text { itrop } \\ \exp \left(-\left(\left(p_{i}-p_{j}\right) / 100\right)^{2}\right) ; i, j>\text { itrop }\end{cases}
$$

We further assume that there is no correlation among different variables, and the spatial correlation of different variables is determined by the spatial correlation matrix, the final background error covariance matrix $\mathrm{B}$ is:

$$
\mathbf{B}=\left[\begin{array}{ccc}
\mathrm{s}_{c c}^{2} S C & \mathbf{O} & \mathbf{O} \\
\mathbf{O} & \mathrm{s}_{l w}^{2} S C & \mathbf{O} \\
\mathbf{O} & \mathbf{O} & \mathrm{s}_{i w}^{2} S C
\end{array}\right]
$$

where $\mathbf{S}_{c c}, \mathbf{S}_{l w}$ and $\mathbf{S}_{i w}$ are the standard deviation of cloud cover, cloud liquid water and cloud ice water content.

\section{Experiment Results}

In this paper, we judge the cloud type by the height (pressure level) of clouds. The height of low clouds are usually lower than 2000 meters (about $800 \mathrm{hPa}$ ), the high 
clouds are located in the height above 6000 meters (about $500 \mathrm{hPa}$ ), and the middle clouds are located in about $500 \mathrm{hPa} \sim 800 \mathrm{hPa}$. We select the brightness temperature of HIR and AMSU-A channels to form a joint observation data set, and meanwhile choose several typical cloud and rain affected atmospheric profiles to analyze.

In Table 1, for profile 1, the cloud covers of three types are not large. The low-middle cloud amount is more than high cloud in profile 2 . The low-middle cloud cover and high cloud cover have not been much difference in profile 3 . The high cloud amount of profile 4 is significantly more than low cloud cover.
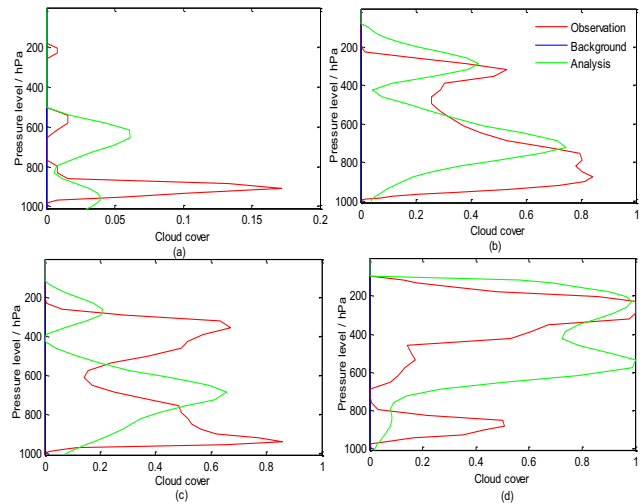

Figue 1. The vertical distribution of cloud amount of different cloud types in profile 1 (a), profile 2 (b), profile 3 (c) and profile 4 (d). Observational cloud cover (red line), background cloud cover (blue line), 1D-Var analysis cloud cover (green line).

The vertical distribution of observational cloud amount (red) of different cloud types in each profile can be seen from Fig. 1. The figure can reflect the vertical distribution of cloud more intuitionistic.

Table1. The cloud cover in each profile

\begin{tabular}{|c|c|c|c|c|c|c|c|}
\hline Profile & \multicolumn{7}{|c|}{ Cloud cover and cloud height } \\
\hline & $\begin{array}{l}\text { Low cloud } \\
\text { cover }\end{array}$ & Low & $\begin{array}{l}\text { cloud } \\
\text { height } \\
(h P a)\end{array}$ & $\begin{array}{l}\text { Middle cloud } \\
\text { cover }\end{array}$ & $\begin{array}{c}\text { Middle cloud } \\
\text { height }(\mathrm{hPa})\end{array}$ & $\begin{array}{c}\text { High cloud } \\
\text { cover }\end{array}$ & $\begin{array}{l}\text { High cloud } \\
\text { height }(\mathrm{hPa})\end{array}$ \\
\hline 1 & 0.1719 & 933.1 & & 0.0156 & 539.7 & 0.0078 & 202.2 \\
\hline 2 & 0.8438 & 875.1 & & 0.8047 & 788.7 & 0.5312 & 318.4 \\
\hline 3 & 0.8594 & 943 & & 0.4922 & 791.7 & 0.6719 & 352.2 \\
\hline 4 & 0.5078 & 882.9 & & 0.1719 & 538 & 1 & 228.8 \\
\hline
\end{tabular}
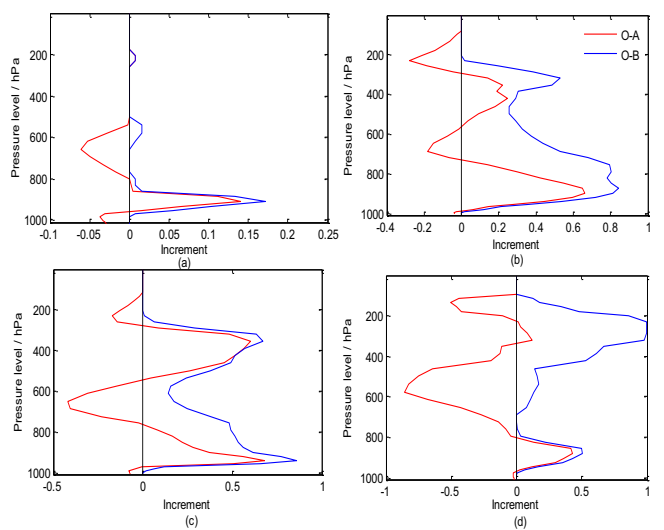

Figure 2. The vertical distribution of cloud amount of different cloud types in profile 1 (a), profile 2 (b), profile 3 (c) and profile $4(\mathrm{~d})$. Analysis increment (observation minus analysis, red line), background increment (observation minus background, blue line).
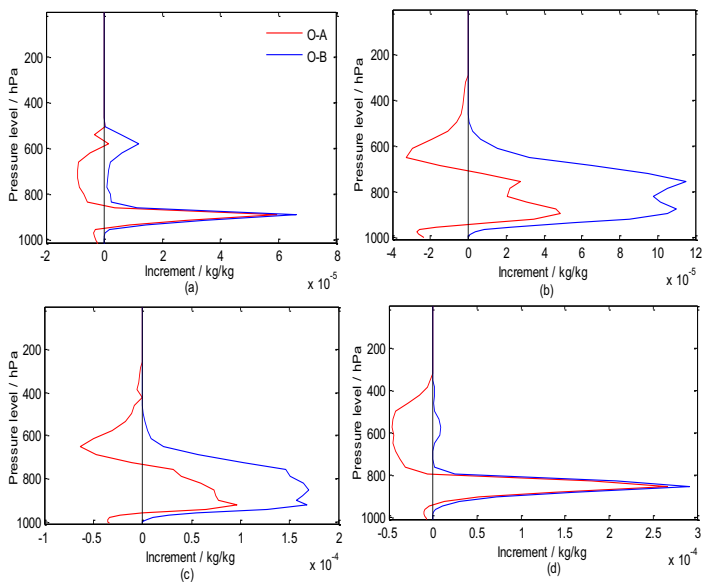

Figure 3. The vertical distribution of increment of cloud liquid water amount in profile 1 (a), profile $2(\mathrm{~b})$, profile 3 (c) and profile 4 (d). Analysis increment (observation minus analysis, red line), background increment (observation minus background, blue line).

Compared to the coarsely initial background estimation of cloud cover, 1D-Var analysis can be closer to the observation, especially when the cloud base is 
relatively high and the cloud amount is relatively small (Fig. 1(d)). However, if the cloud base is too low and the cloud cover is too large (Fig. 1b, 1c), false bias field may be produced, for instance, the low cloud leads to the total cloud cover field to be distorted though the cloud covers are all not large in Fig. 1(a). From the analysis incremental (observation minus analysis, red line) in Fig. 2 , It can also be seen that the $1 \mathrm{D}-\mathrm{Var}$ retrieved effect is better where the high cloud exists.
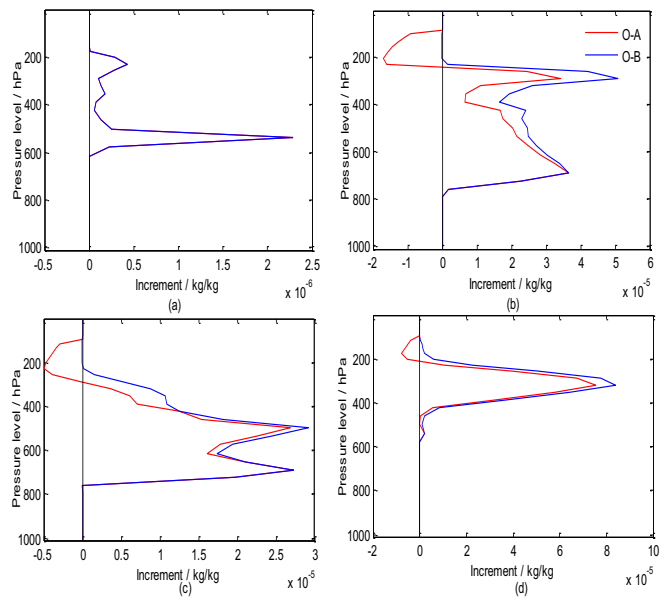

Figure 4. The vertical distribution of the increment of cloud ice water amount in profile 1 (a), profile 2 (b), profile 3 (c), profile 4 (d). Analysis increment (observation minus analysis, red line), background increment (observation minus background, blue line).

Fig. 3 and Fig. 4 show the vertical distribution of incremental cloud liquid water and cloud ice water, respectively. The height of cloud water is generally lower than 600hpa, the analysis and background increment have no much difference different when the cloud amount is small (Fig. 3(a)). However, 1D-Var can significantly improve the quality of cloud liquid water when the low cloud cover becomes big (Fig. 3(b), 3(c)). For ice cloud, the increments are close in the lower atmosphere because ice cloud usually distributes high in the atmosphere. It is clear that they separate from each other above $400 \mathrm{hPa}$, the main reason may be that the water vapor reduces and scattering effect increases along with the altitude becomes higher.

\section{Conclusions}

Built on the 1D-Var algorithm, we investigated the retrieved effect of three cloud water variables (cloud cover, cloud liquid water and cloud ice water) in atmospheric field. The results of the experiment show that the performance of 1D-Var system works well and can obtain good estimation of cloud cover when the cloud amount is small, however, the retrieved cloud amount is not too ideal when the cloud cover is large or the cloud height is too low. 1D-Var has an overall reformative effect on cloud liquid water and cloud ice water. But due to the strongly physical nonlinearity in cloud and rain, the observed operator may not be able to describe the physical process accurately. Next, we will estimate more precise background and its error covariance of cloud water variables to obtain a better inversion effect.

\section{Acknowledgment}

The work was supported by the Natural Science Foundation of China (Grant No. 41375113).

\section{References}

[1] F. Chevallier and P. Bauer, "Model Rain and Clouds over Oceans: Comparison with SSM/I Observations," Monthly Weather Review, vol. 131, 2003, pp. 1240-1255.

[2] G. Ma, Z. Y. Fang and F. Y. Zhang, "The Impact of Cloud Parameters on the Simulated Errors in RTTOV5," Quaterly Journal of Applied Meteorology, vol. 12, 2001, pp. 385-392.

[3] W. Y. Ding, Q. L. Wan, Z. T. Chen, Y. Y. Huang and C. Z. Zhang, "Assimilation of HIRS/3 Brightness Temperature in Cloud Condition and Its Impact to CHANCHU Typhoon", Acta Meteorologica Sinica, vol. 68, 2010, pp. 70-78.

[4] Y. L. Liu, "Application Research of 1D-Var Retrieval Technology in Cloud- and Precipitation effected Satellite Microwave Observation Data Assimilation", Changsha: National University of Defense Technology, 2013.

[5] Q. B. Huang, "A Variational Data Assimilation Technology of the Cloud and Rain Affected Satellite Microwave Data", Changsha: National University of Defense Technology, 2011.

[6] Q. B. Huang, X.. Q. Cao, M. B. Zhu, W. M. Zhang and B. N. Liu, "New data assimilation system DNDAS for high-dimensional models", Chin. Phys. B, vol. 25, 2016, pp. 50502-050502. 